\title{
Host-based Intrusion Detection System (HIDS)
}

\author{
AmanYadav, Abhishek Srivastav, Abhinandan Tiwari, Krishna Vir Singh
}

\begin{abstract}
This paper presents the data analysis and feature extraction of KDD dataset of 1999. This is used to detect signature based and anomaly attacks on a system. The process is supported by data extraction as well as data cleaning of the above mentioned data set. The dataset consists of 42 parameters and 58 services. These parameters are further filtered to extract useful attributes. Every attack in the dataset is labeled either with "normal" or into four different attack types i.e. denial-of-service, network probe, remote-to-local or user-to-root. Using different machine learning algorithms, the work tries to compare the individual accuracy, True Positive and False positive rate of every algorithm with every other algorithm. The work focuses its attention to increase security through detection of static as well as dynamic attack.

Keywords: Host based intrusion detection, Data cleaning, Data analysis, Machine learning, KDD cupp'99, Attack, anomaly.
\end{abstract}

\section{INTRODUCTION}

The paper talk about the implementation and importance of a host based intrusion detection system. This is done using refining and selection of importance features of given dataset which is achieved using data cleaning and data mining operation performed on this dataset. Some attributes contain redundant information, while some other contain false correlations; either type of these can hinder the results. Traffic reduction is also one of the important aspects of the work. Traffic reduction can be achieved by using filters prior to network data collection. Filters ignore certain type of traffic thus reducing the traffic. Filters should be used with almost care as it can also filter or ignore some important parameters or network necessary for the determination of an attack on the host. This all data has been inculcated in the KDD dataset.All the attacks mentioned in KDD Cup ' 99 can be classified in any of the four attacks i.e. Denial of Service, REMOTE TO LOCAL, USER TO ROOT and PROBING.

\section{A. Denial of Service}

In this type of cyber-attack, attacker restricts the genuine users from accessing the services of the host which is attacked. The attack of this kind is performed by sending the huge amount of requests to the host thus making it overwhelmed and may lead to a complete crash in some cases.

Revised Manuscript Received on June 15, 2020.

* Correspondence Author

AmanYadav*, CSE Department, ABESEC Ghaziabad AKTU Lucknow , India. aman.16bcs2046@abes.ac.in.

AbhishekSrivastav, CSE Department, ABESEC Ghaziabad AKTU Lucknow, India. abhishek.16bcs2035@abes.ac.in.

AbhinandanTiwari, CSE Department, ABESEC Ghaziabad AKTU Lucknow, India. abhinandan.16bcs2051@abes.ac.in.

Krishna Vir Singh, CSE Department, ABESEC Ghaziabad AKTU Lucknow , India. krishnavir.singh@abes.ac.in.

(C) The Authors. Published by Blue Eyes Intelligence Engineering and Sciences Publication (BEIESP). This is an open access article under the CC BY-NC-ND license (http://creativecommons.org/licenses/by-nc-nd/4.0/)

\section{B. Remote To Local}

This kind of attack is generally performed by the attacker in order to gain illegitimate access to the targeted machine so that the attacker could steal or manipulate the data also the Attacker could inject the viruses or any kind of malicious files.

\section{User To Root}

In $u 2 r$ attack the attacker first tries to get access to victim machine as normal user then tries to gain the access to root level after it gains the access as a root level the whole security of the targeted system is compromised and the attacker then can exploit the system at root level.

\section{Probing}

In this the attacker performs the scan of the entire network to find the vulnerabilities in order to find the weak points so as to gain access to the system and its files. This attack is most common because it can be performed with very little technical expertise.

This paper consists of total 22 attacks, every one of which lies in any of the four attack types e.g. pod attack is a type of DoS attack, guess_passwd attack is a R2L attack, buffer_overflow

\section{MOTIVATION}

Till date a lot of work has been done in the field of security but still there is a lot of scope for its improvement. No system with $100 \%$ security has been designed, there are some security flaws in every system added by all the attacks and intrusion attempt are not yet known. Tons of virus and malware are being generated everyday attacking other tons of system.

Some Recent Attacks On Indian Facilities Or On Indian Users: -

1. Agent Smith Malware in India (Aug. 2019).

2. Malware Attack on Kudankulam Nuclear Power plant (Dec. 2019).

3. WhatsApp hacking of Indian Journalist by Israeli made spyware (Nov. 2019).

4. Alert Issued by Ministry of Home against Stranghogg Bugg (Jan. 2020).

Increasing number of cyber-attacks has increased our keen interest towards intrusion and different ways in which we can tackle them.

\section{SCOPE OF HIDS}

Seeing the present day scenarios intrusion attacks become a quite obvious and quite real thread for a small scale company to a large scale companies. In fact the most expanding field for now is cyber space and with its expansion the risk of attacks expands as well.

\section{Published By:}




\section{Host-based Intrusion Detection System (HIDS)}

Nowadays even nations are involved in $5^{\text {th }}$ generation warfare, a classic example being the latest cyber-attack carried by USA on IRAN. Thus this explains the need of HIDS in not just in companies but also for national security.

\section{RELATED WORK}

Unfortunately, KDD dataset of 1999 is the best dataset available for host based intrusion detection system. Every traffic in dataset is either "normal" or any one of the four attack types. Four attacks type being denial-of-service (dos), network probe (probe), remote-to-local (r2l) and user-to-root (u2r) attacks. The data even contain anomalous behaviours of normal users acting like privileged users.
The main aim of perform a dos attack is to prevent user to access a service e.g. 'TCP syn floods'. Probe attacks like 'ipsweeps' are used to collect information about the targets. The attackers performing r2l attack try to gain admin control over machine e.g. 'dictionary attack'. In u2r attack the user with user access tries to gain privileged access. Different types of buffer overflow attacks lie in this category. Attackers can even combine different type of attack to increase their operational limits. In most of the combine attacks case the attackers go for probe $\rightarrow$ r2l $\rightarrow$ u2r pattern. Some attackers combine different attack to hide the main motive behind some other attack. For an instance many attackers perform dos attack and r2l attack, so that the user gives its attention on dos attack and r2l attack can be performed without and hindrance.

Table- I: The dataset contains 41 features along with their description

\begin{tabular}{|c|c|c|}
\hline Nr & Name & $\begin{array}{l}\text { Features } \\
\text { Description }\end{array}$ \\
\hline 1 & duration & duration of connection in seconds \\
\hline 2 & protocol_type & connection protocol (tep, udp, iemp) \\
\hline 3 & service & dst port mapped to service (e-g. http, ftp, ..) \\
\hline 4 & flag & normal or error status flag of connection \\
\hline $\mathbf{5}$ & sre-bytes & number of data bytes from sre to dst \\
\hline 6 & dst_bytes & bytes from dst to sre \\
\hline 7 & land & 1 if connection is from/to the same host/port; else 0 \\
\hline 8 & wrong_fragment & number of 'wrong' fragments (values $0,1,3$ ) \\
\hline 9 & urgent & number of urgent packets \\
\hline 10 & hot & number of 'hot' indicators (bro-ids feature) \\
\hline 11 & num_failed_logins & number of failed login attempts \\
\hline 12 & logged in & 1 if successfully logged in; else 0 \\
\hline 13 & num_compromised & number of 'compromised' conditions \\
\hline 14 & root shell & 1 if root shell is obtained; else 0 \\
\hline 15 & su_attempted & 1 if 'su root' command attempted; else 0 \\
\hline 16 & num_root & number of 'root' acoesses \\
\hline 17 & num_file_ereations & number of file creation operations \\
\hline 18 & num_shells & number of shell prompts \\
\hline 19 & num_access files & number of operations on access control files \\
\hline 20 & num_outbound_cmas & number of outbound commands in an ftp session \\
\hline 21 & is hot login & 1 if login belongs to 'hot' list (e.g. root, adm); else 0 \\
\hline 22 & is_guest_login & 1 if login is 'guest' login (e.g. guest, anonymous); else 0 \\
\hline 23 & count & $\begin{array}{l}\text { number of connections to same host as current } \\
\text { connection in past two seconds }\end{array}$ \\
\hline 24 & srv_count & $\begin{array}{l}\text { number of connections to same service as current } \\
\text { connection in past two seconds }\end{array}$ \\
\hline 25 & serror_rate & $\%$ of connections that have 'SYN' errors \\
\hline 26 & srv_serror_rate & \% of connections that have 'SYN' errors \\
\hline 27 & rerror_rate & \% of connections that have 'RE.J' errors \\
\hline 28 & srv_rerror_rate & \% of connections that have 'REJ' errors \\
\hline 29 & same_srv_rate & \% of connections to the same service \\
\hline 30 & diff_srv_rate & $\%$ of connections to different services \\
\hline 31 & srv_diff_host_rate & $\%$ of connections to different hosts \\
\hline 32 & dst_host_count & count of connections having same dst host \\
\hline 33 & dst_host_srv_count & $\begin{array}{l}\text { count of connections having same dst host and } \\
\text { using same service }\end{array}$ \\
\hline 34 & dst_host_same_srv_rate & $\begin{array}{l}\text { \% of connections having same dst port and } \\
\text { using same service }\end{array}$ \\
\hline 35 & dst host_diff_srv_rate & \% of different services on current host \\
\hline 36 & dst_host_same_src_port rate & \% of connections to current host. having same sre port. \\
\hline 37 & dst_host_srv_diff host_rate & $\%$ of connections to same service coming from diff. hosts \\
\hline 38 & dst_host_serror_rate & $\%$ of connections to current host that have an So error \\
\hline 39 & dst host_srv_serror rate & $\begin{array}{l}\text { \% of connections to current host and specified service } \\
\text { that have an So error }\end{array}$ \\
\hline 40 & dst_host_rerror_rate & $\%$ of connections to current host that have an RST error \\
\hline 41 & dst_host_srv_rerror_rate & $\begin{array}{l}\text { \% of connections to the current host and specified service } \\
\text { that have an RST error }\end{array}$ \\
\hline 42 & connection.type & \\
\hline
\end{tabular}

There are basically three methodology or neural networks that are used in implementation of host based intrusion detection system: -

\section{A. Recurrent Neural Network}

It is a form of network with backward connection. The output layer in the network is fed back into either that layer or the previous layer in the network.

\section{B. Convolutional Neural Network}

It is primarily used in image processing. It is used to detect patters. This network helps in the identification of attack patterns or change in a normal pattern.

\section{Sequence anomaly detection using language modeling}

In this system call is represented as integers (1 to 340). We can estimate probability of sequence occurring using probability distribution.

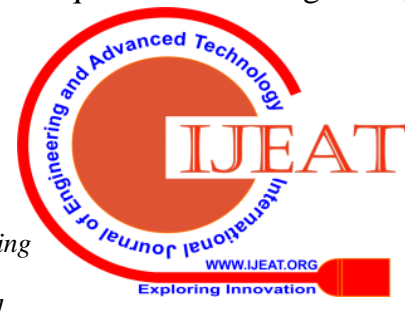


This network helps in estimation of attack based on the sys logs and pattern analysis. The KDD Cup '99 gives us idea about some errors as well. The errors include syn error, rej error, S0 errorandrst error.

Table- II: Different attacks that are classified under the four attack categories

\begin{tabular}{l|l|l|l}
\hline \multicolumn{1}{c|}{$\begin{array}{c}\text { Name of the } \\
\text { attack }\end{array}$} & Type & \multicolumn{1}{c}{ Mechanism } & \multicolumn{1}{c}{ Effect of the attack } \\
\hline back & DoS & Abuse/Bug & Slows down server response \\
\hline land & DoS & Bug & Slows down server response \\
\hline neptune & DoS & Abuse & Slows down server response \\
\hline smurf & DoS & Abuse & Slows down the network \\
\hline pod & DoS & Abuse & Slows down server response \\
\hline teardrop & DoS & Bug & Reboots the machine \\
\hline loadmodule & U2R & Poor environment sanitation & Gains root shell \\
\hline buffer_overflow & U2R & Abuse & Gains root shell \\
\hline rootkit & U2R & Abuse & Gains root shell \\
\hline perl & U2R & Poor environment sanitation & Gains root shell \\
\hline phf & R2L & Bug & Executes commands as root \\
\hline guess_passwd & R2L & Login misconfiguration & Gains user access \\
\hline warezmaster & R2L & Abuse & Gains user access \\
\hline imap & R2L & Bug & Gains root access \\
\hline multihop & R2L & Abuse & Gains root access \\
\hline ftp_write & R2L & Misconfiguration & Gains user access \\
\hline spy & R2L & Abuse & Gains user access \\
\hline warezclient & R2L & Abuse & Gains user access \\
\hline satan & Probe & Abuse of feature & Looks for known vulnerabilities \\
\hline nmap & Probe & Abuse of feature & Identifies active ports on a machine \\
\hline portsweep & Probe & Abuse of feature & Identifies active ports on a machine \\
\hline ipsweep & Probe & Abuse of feature & Identifies active machines \\
\hline & &
\end{tabular}

Massachusetts Institute of Technology Lincoln labs gave KDD dataset, which is found to be very helpful for framing any machine learning model we in our project have used four machine learning algorithms for classifying various attacks into 4 broad categories. Each of the machine learning algorithms has its own accuracy for each type of categories. The statistical information present in the dataset contains sufficient amount of instances for each type of attacks.It makes very easy to divide the training and testing dataset.

\section{IMPLEMENTATION AND RESULT}

\section{A. Machine Learning Approach}

Approach of machine learning is very simple in a machine learning we try to design a system that is able to complete the task by closely studying the training dataset The formed predictive model then based upon the findings during training dataset classifies the test data into various labels. Accuracy and reliability of the predictive model increases with the increase in the amount of data present and this demand is fulfilled by the KDD dataset. Thus it makes very obvious to follow the machine learning approach.

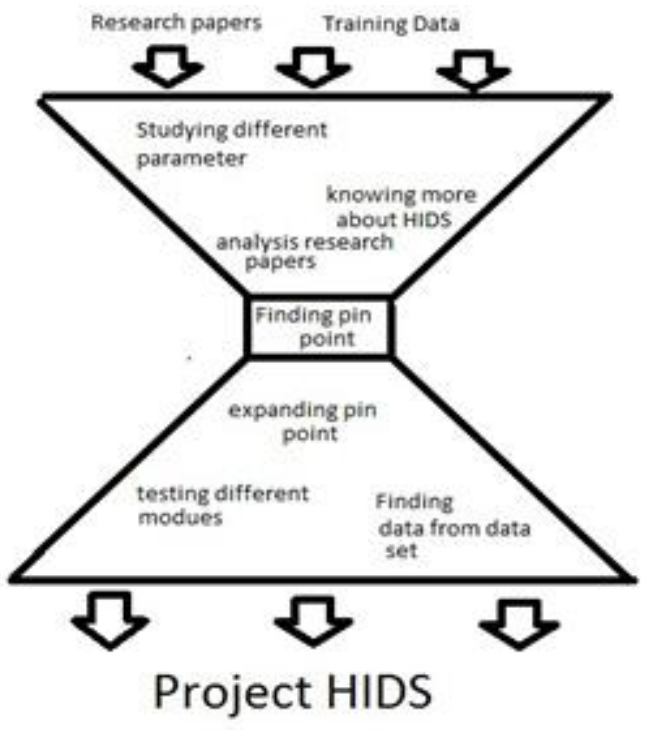

Fig.1. Anomaly detection flowchart using machine learning algorithms

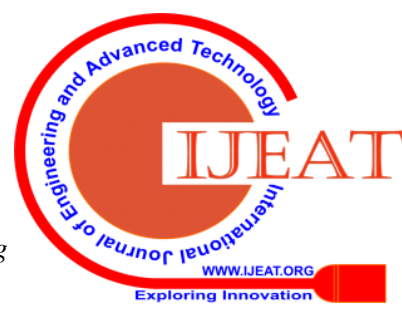




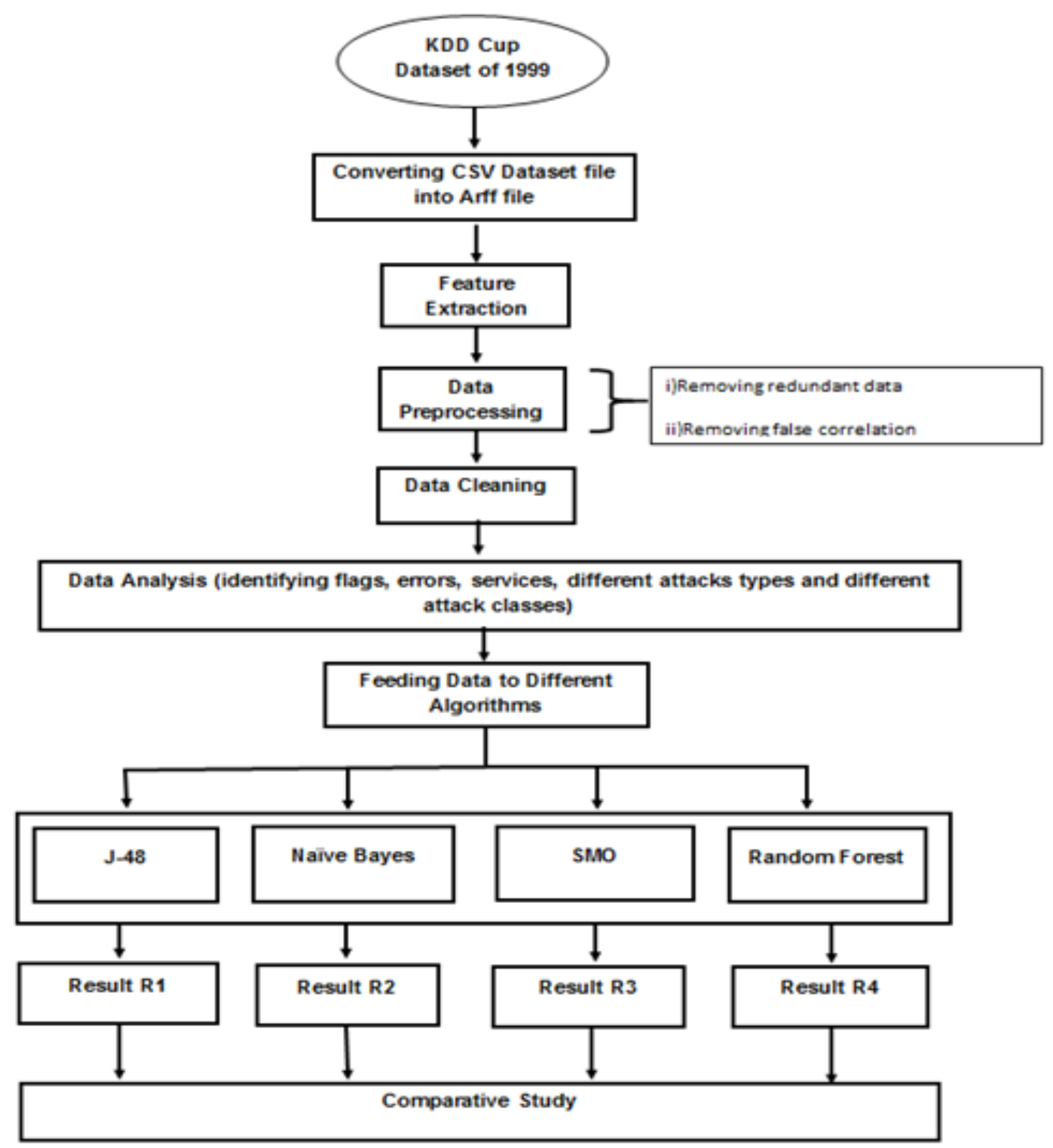

Fig.2. the above flowchart clearly illustrates the steps required to accomplish the task.

\section{B. Comparative study (Accuracy of different Algorithms)}

The paper involves use of four algorithms i.e. Naïve Bayes, J-48, Random forest and SOM algorithm. Each algorithm generates result output with different accuracy and these accuracies are compared in order understand

the efficiency of every algorithm with KDD Cup '99 datasets. The output generated contains the TP rate and FP rate. TP rate stands for true positive rate and it measures the actual positives that are correctly identified. The other name for TP rate is "sensitivity" .FP rate stands for false positive rate and it is given by following expression: -

$$
\begin{aligned}
& (\mathrm{FP} / \mathrm{N})=\mathrm{FP} /(\mathrm{FP}+\mathrm{TN}) \\
& \mathrm{N}=\mathrm{FP}+\mathrm{TN}
\end{aligned}
$$

Where FP is the number of false positives, $\mathrm{TN}$ is the number of true negatives and $\mathrm{N}$ is the total number of negatives. 
Table-III: TP rate and FP rate of algorithms for every attack level

\begin{tabular}{|c|c|c|c|c|c|c|c|c|}
\hline \multirow[t]{2}{*}{ Class } & \multicolumn{2}{|c|}{ NaiveBayes } & \multicolumn{2}{|c|}{148} & \multicolumn{2}{|c|}{ Random Forest } & \multicolumn{2}{|c|}{ SOM } \\
\hline & TP Rate & FP Rate & TP Rate & FP Rate & TP Rate & FP Rate & TP Rate & FP Rate \\
\hline Normal & 71.2 & 0.3 & 99.9 & 1 & 100 & 0.8 & 99.9 & 2.6 \\
\hline buffer overflow & 80 & 0.9 & 70 & & 86.7 & 0 & 53.3 & 0 \\
\hline loadmodule & 33.3 & 0.1 & 0 & 0 & 22.2 & 0 & 0 & 0 \\
\hline perl & 33.3 & 0 & 0 & 0 & 66.7 & 0 & 33.3 & 0 \\
\hline neptune & 98.6 & 0.1 & 99.9 & 0 & 100 & 0 & 100 & 0 \\
\hline smurf & 99 & 6.6 & 100 & 0 & 100 & 0 & 91.1 & 0 \\
\hline guess passwd & 94.3 & 0.1 & 94.3 & 0 & 96.2 & 0 & 96.2 & 0 \\
\hline pod & 98.9 & 0.6 & 98.9 & 0 & 98.9 & 0 & 98.9 & 0 \\
\hline teardrop & 100 & 0 & 100 & o & 100 & 0 & 99.4 & 0 \\
\hline portsweep & 44.9 & 0.27 & 97.7 & 0 & 96.7 & 0 & 97.2 & 0 \\
\hline ipsweep & 97.4 & 5 & 97.9 & 0 & 96.7 & 0 & 97.4 & 0.1 \\
\hline land & 94.4 & 0 & 83.3 & 0 & 94.4 & 0 & 100 & 0 \\
\hline ftp_write & 50 & 0.2 & 0 & 0 & so & 0 & 12.5 & 0 \\
\hline back & 98.4 & 5.7 & 99.2 & 0 & 100 & 0 & 98.8 & 0 \\
\hline imap & 91.7 & 0 & 25 & 0 & 91.7 & 0 & 83.3 & 0 \\
\hline satan & 84 & 1 & 96.2 & 0 & 95.1 & 0 & 89.2 & 0 \\
\hline phf & 100 & 0.7 & 100 & 0 & 100 & 0 & 0 & 0 \\
\hline nmap & 31.7 & 0.7 & 93.1 & 0 & 96.6 & 0 & 32.4 & 0 \\
\hline multihop & 28.6 & 0 & 28.6 & 0 & 28.6 & 0 & 0 & 0 \\
\hline warezmaster & 80 & 0 & 75 & 0 & 85 & 0 & 75 & 0 \\
\hline warezclient & 49.8 & 2.8 & 98.6 & 0 & 98.8 & 0 & 91.6 & 0 \\
\hline spy & 100 & 0 & 0 & 0 & 0 & 0 & 0 & 0 \\
\hline rootkit & 30 & 0.3 & 0 & 0.9 & 10 & 0.7 & 0 & 0 \\
\hline
\end{tabular}

algorithms (Random Forest, SMO, Naïve Bayes, J48), it has

The classifier values are different for all the four algorithms i.e. correctly classified instances, incorrectly classified instances, mean absolute error, root mean squared error, relative absolute error, root relative squared error and total numbers of instances. After applying various classifying been found that the Random Forest algorithm has been successful in detecting various attacks in KDD Dataset and classifying instances of KDD dataset correctly.

Table-IV: Classifier values for different algorithms

\begin{tabular}{|c|c|c|c|c|c|c|c|c|}
\hline \multirow{2}{*}{$\begin{array}{l}\text { Classifier } \\
\text { Correctly Classified Instances }\end{array}$} & \multicolumn{2}{|c|}{ Naive Bayes } & \multicolumn{2}{|c|}{$J 48$} & \multicolumn{2}{|c|}{ Random Forest } & \multicolumn{2}{|c|}{ SOM } \\
\hline & 89739 & $99.80 \%$ & 65655 & $73.0165 \%$ & 89794 & 99.8621 d & 89455 & $99.4851 \%$ \\
\hline Incorrectly Classified Instances & 179 & $0.20 \%$ & 24263 & $26.9835 \%$ & 124 & $0.1379 \%$ & 463 & $0.5149 \%$ \\
\hline Mean absolute error & \multicolumn{2}{|c|}{0.0002} & \multicolumn{2}{|c|}{0.0232} & \multicolumn{2}{|c|}{0.0003} & \multicolumn{2}{|c|}{0.0794} \\
\hline Root mean squared error & \multicolumn{2}{|c|}{0.0125} & \multicolumn{2}{|c|}{0.1469} & \multicolumn{2}{|c|}{0.0099} & \multicolumn{2}{|c|}{0.1961} \\
\hline Relative absolute error & \multicolumn{2}{|c|}{$1.42 \%$} & \multicolumn{2}{|c|}{$143.81 \%$} & \multicolumn{2}{|c|}{$1.81 \%$} & \multicolumn{2}{|c|}{$492.51 \%$} \\
\hline Root relative squared error & \multicolumn{2}{|c|}{$13.90 \%$} & \multicolumn{2}{|c|}{$163.69 \%$} & \multicolumn{2}{|c|}{$11.04 \%$} & \multicolumn{2}{|c|}{$218.59 \%$} \\
\hline Total Number of Instances & \multicolumn{2}{|c|}{89918} & \multicolumn{2}{|c|}{89918} & & 918 & \multicolumn{2}{|c|}{89918} \\
\hline
\end{tabular}

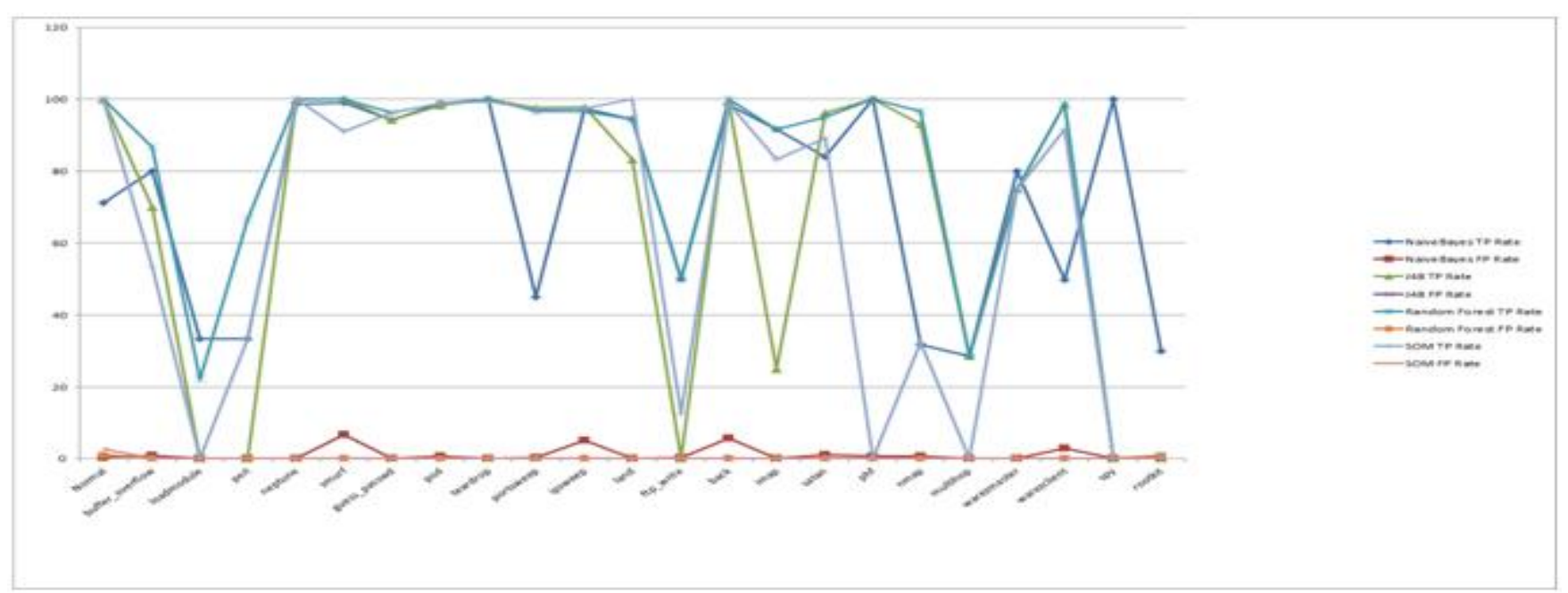

Fig. 3. The below graph represent FP and TP rate for different algorithms on different attacks

Retrieval Number: E9903069520/2020 CBEIESP DOI: 10.35940/ijeat.E9903.069520

Journal Website: www.ijeat.org
Published By:

Blue Eyes Intelligence Engineering

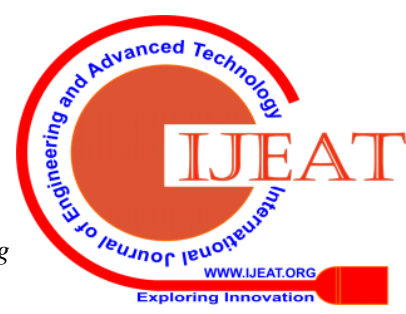

(C) Copyright: All rights reserved. 

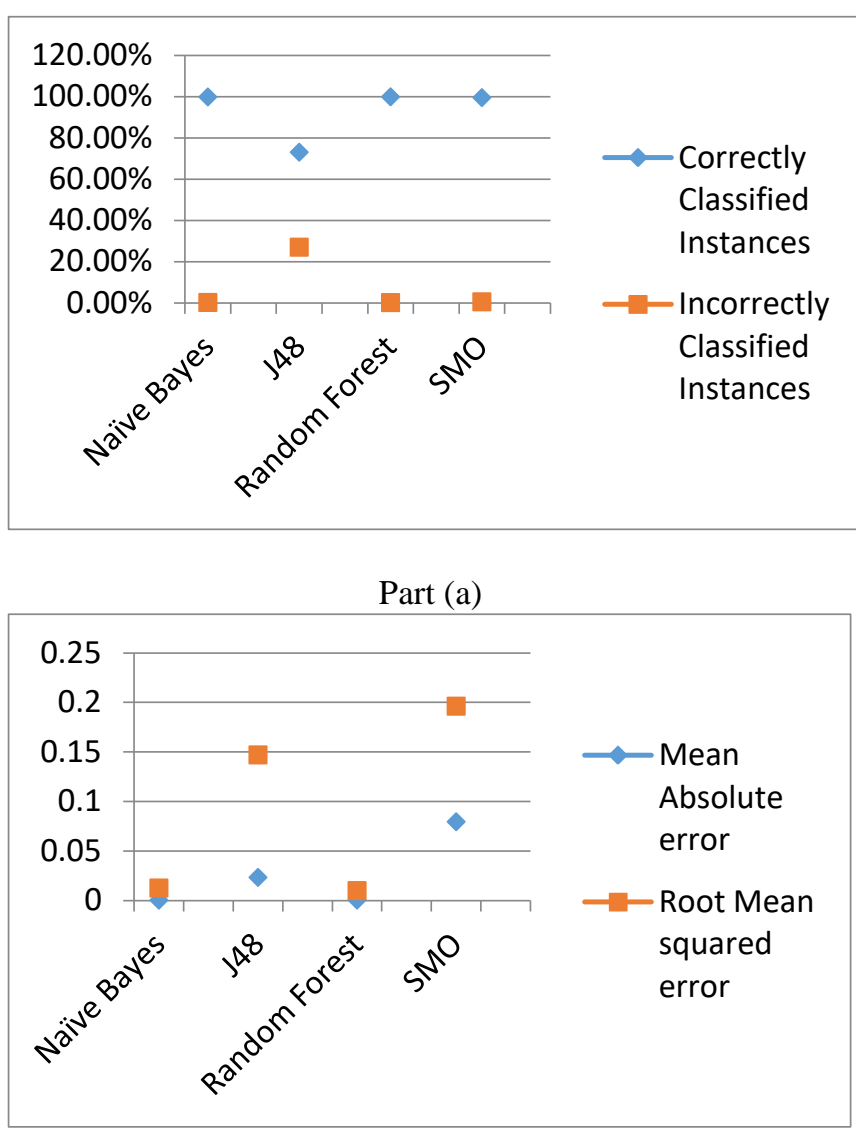

Part (b)

The classifier values and errors for different algorithms represented in form of graph

\section{CONCLUSION AND FUTURE SCOPE}

Because of the acute demand of an effectual HIDS in the security related to the network, many researchers are trying very hard and are working for better approach that can lead to better results. In this research paper depicts the usefulness of the KDD dataset for testing various classifiers. Study focuses on preprocessing of KDD Dataset and a lot of work has been done to remove all sorts of factors that can lead to bias results. Originally KDD has 42 attributes and after studying all of them in detail and finding which of them are required for HIDS we have reduced them to 31 parameters. Now talking about the future scope there is a lot work which is need to be done to increase the efficiency of the classifying algorithms we can tune the algorithms we can alter the $\%$ of training and testing data. We need to increase the efficiency of the HIDS.

\section{REFERENCES}

1. Staudemeyer, Ralf C., and Christian W. Omlin. "Extracting salient features for network intrusion detection using machine learning methods." South African computer journal 52.1 (2014): 82-96.

2. Akbar, Shaik, K. NageswaraRao, and J. A. Chandulal. "Intrusion detection system methodologies based on data analysis." International Journal of Computer Applications 5.2 (2010): 10-20.

3. Win, MyaThidarMyo, and KyawThetKhaing. "Detection and Classification of Attacks in Unauthorized Accesses." International Conference on Advances in Engineering and Technology (ICAET'2014). 2014.

4. Sabhnani, Maheshkumar, and GürselSerpen. "KDD Feature Set Complaint Heuristic Rules for R2L Attack Detection." Security and Management. 2003.

5. Chawla A, Lee B, Fallon S, Jacob P. Host based intrusion detection system with combined CNN/RNN model. InJoint European Conference on Machine Learning and Knowledge Discovery in Databases 2018 Sep 10 (pp. 149-158). Springer, Cham.

6. Bace RG. Intrusion detection. Sams Publishing; 2000.

7. Olusola, A.A., Oladele, A.S. and Abosede, D.O., 2010, October. Analysis of KDD'99 intrusion detection dataset for selection of relevance features. In Proceedings of the world congress on engineering and computer science (Vol. 1, pp. 20-22). WCECS.

8. Aggarwal P, Sharma SK. Analysis of KDD dataset attributes-class wise for intrusion detection. Procedia Computer Science. 2015 Jan $1 ; 57: 842-51$

9. Lee, J.H., Lee, J.H., Sohn, S.G., Ryu, J.H. and Chung, T.M., 2008, February. Effective value of decision tree with KDD 99 intrusion detection datasets for intrusion detection system. In 2008 10th International Conference on Advanced Communication Technology (Vol. 2, pp. 1170-1175). IEEE.

10. Chae HS, Jo BO, Choi SH, Park TK. Feature selection for intrusion detection using NSL-KDD. Recent advances in computer science. 2013 Nov:184-7.

11. Lakhina, Shilpa, Sini Joseph, and BhupendraVerma. "Feature reduction using principal component analysis for effective anomalybased intrusion detection on NSL-KDD." (2010).

\section{AUTHOR PROFILE}

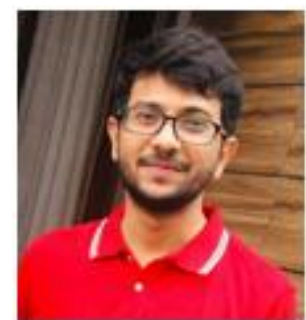

Mr. Aman Yadav is pursuing his B.Tech in Computer Science and Engineering from ABES Engineering College, Ghaziabad which is affiliated to AKTU. He has completed his 10th and 12th from Mercy Memorial School, Kanpur affiliated to ICSE. He has worked in java based technologies and J2EE technologies. $\mathrm{He}$ is currently working in the domain of cyber security and computer networking. He is very eager to learn new technologies because it further expands the opportunities upon which he can work upon and solve the problems. He believes that different technologies can be brought together in order to make a product reliable.

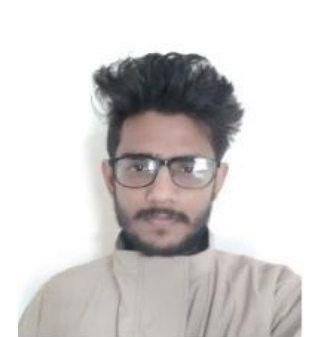

Mr. Abhishek Srivastav from Gorakhpur city of Uttar Pradesh. My family which includes his mother, father and sister have always provide their support in his journey as a student and as an individual.His father is a government officer and his mother is a home maker. In the way to his graduation from ABES Engineering college in Computer science, he has completed his high school and intermediate from Little Flower School (Salempur) and Sunbeam Bhagwanpur(Varanasi) with 83\% and 93\% respectively.He had a great enthusiasm towards computers that helped him in choosing his path for graduation. Computer and cyber security had always been in Abhshek's mind since he first started using computers. Moving up in his life he had looked forward to expand his knowledge and his work in the field of computers and their security.

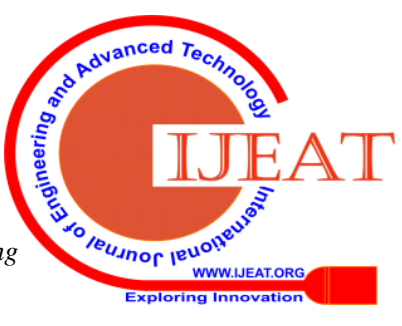




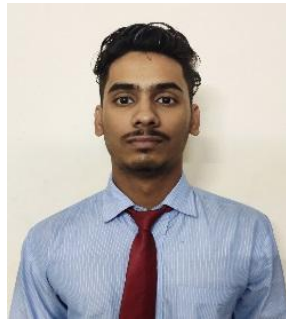

Mr. Abhinandan Tiwari is currently pursuing his B.tech in Computer Science and Engineering from ABES Engineering College which is affiliated to AKTU. He has completed his 10th and 12th from Bright Way College Lucknow with 8.4 CGPA and 83.4\%. He worked on JAVA based technologies and J2EE technologies .He is currently working is the domain of networking and cyber security. Computer and cyber security had always been in my mind since he first started using computers. Moving up in my life i look forward to expand my knowledge and my work in the field of computer networking and cyber security.

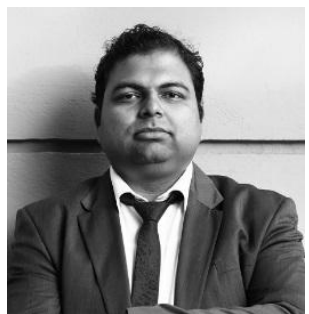

Mr. Krishna Vir Singh is Currently Associated with ABES Engineering College, Ghaziabad as Assistant Professor. He has More Than 11 Years of Experience in Education, Training and Software Development Industry. He is CISCO Certified Instructor for CCNA ( Routing \& Switching). CCNA( Cyber Ops), IoT Fundamentals : Connecting Things, IoT Fundamentals : Big Data Analytics, IoT Security and he is also a ICSI Certified Network Security Specialist. His Research Interests includes Genetic Algorithms, Software Testing, IoT, Network Security \& Cyber Security. He has also filed patent in the area related to IoT.

Published By:

Blue Eyes Intelligence Engineering

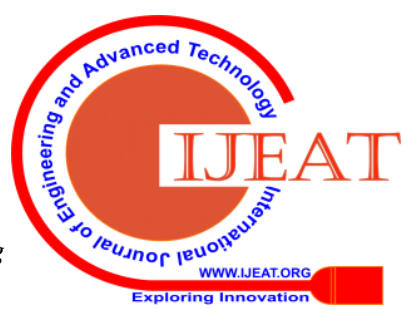

